\title{
Validação de um Programa de Treinamento Simulado de Habilidades Laparoscópicas por Residentes de Cirurgia
}

\author{
Validation of a Simulated Laparoscopic Skills \\ Training Program by Surgical Residents
}

\author{
Ricardo Monteiro de Sá Barreto \\ Hermano Alexandre Lima Rocha ${ }^{I}$ \\ Glaydison Cesar de Oliveira Borges ${ }^{I}$ \\ Arnaldo Aires Peixoto Júnior ${ }^{I}$ \\ Luiz Gonzaga de Moura Júnior \\ Raquel Peixoto ${ }^{I I, I}$
}

\section{PALAVRAS-CHAVE \\ - Treinamento por Simulação. \\ - Laparoscopia. \\ - Internato e Residência.}

${ }^{I}$ Centro Uniersitário Chistus, Fortaleza, Ceará, Brasil.

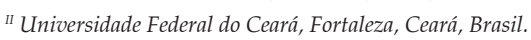

\begin{abstract}
RESUMO
Introdução: Os treinamentos com simuladores para videocirurgia têm sido propostos como ferramentas de ensino, permitindo avaliação formativa de alunos em condições mais controladas e seguras. Objetivo: Validar um programa estruturado de treinamento de endossuturas para residentes de cirurgia, por meio de duas escalas estruturadas de aquisição de habilidades laparoscópicas e da percepção dos participantes. Métodos: Estudo experimental longitudinal quantitativo, que contou com a participação de 12 residentes de cirurgia provenientes de quatro hospitais distintos. O treinamento consistia na confecção de endossuturas com simuladores de vídeo em sete sessões num período de sete meses. A avaliação da progressão da competência técnica dos alunos foi realizada por três avaliadores, utilizando-se o Objective Structured Assessement of Technical Skills (Osats) e a Escala de Progressão de Proficiência (EPP) em Endossuturas. Os residentes usaram um questionário com escala de Likert de 1 a 5 para avaliar o programa de treinamento realizado quanto à aquisição de habilidades específicas. Foram feitos os testes de Anova e Qui-Quadrado para comparação entre variáveis. As correlações e associações entre duas variáveis numéricas foram verificadas por meio de regressão linear simples e múltipla, quando foram incluídos os fatores determinantes além do número de sessões de treinamento. Foi calculado o ranking médio para análise da escala de Likert. Foram consideradas significativas as comparações com valor de $p \leq 0,05$. Este estudo foi aprovado pelo Comitê de Ética em Pesquisa da instituição. Resultados: Quanto ao desempenho dos residentes observado pelos avaliadores por meio do Osats, observou-se melhora estatisticamente significante na pontuação em relação aos itens: respeito ao tecido $(p=0,006)$, tempo e movimento $(p=0,001)$, conhecimento e manuseio dos instrumentos ( $p=0,002)$, fluxo da cirurgia ( $p=<0,001)$, conhecimento do procedimento específico ( $p$ $=0,001$ ), pelo teste Qui-Quadrado. Em relação ao número total de pontos pela EPP e à nota média nos testes pelo Osats modificado, o total e a nota média nos testes foram significantes $(<0,001 e<0,001)$, utilizando-se o teste Kruskal-Wallis ao longo dos meses de treinamento. Na percepção dos alunos, o programa de treinamento contribuiu para o desenvolvimento de habilidades cirúrgicas, tendo como itens mais bem avaliados o manuseio de pinças e porta-agulhas e a confecção de nós. Os itens mais mal avaliados foram a hapticidade e a passada de alça. Demonstrou-se ganho significativo na aquisição de competência técnica pelos residentes durante o curso, percebido por meio dos dois instrumentos de avaliação utilizados (Osats e EPP). Conclusão: Houve ganho significativo de habilidades pelos residentes de cirurgia ao longo dos sete meses do programa de treinamento simulado, demonstrado pelos dois instrumentos de avaliação estruturada e pela percepção dos residentes.
\end{abstract}




\section{KEY-WORDS}

- Simulation Training.

- Laparoscopy.

- Internship and Residency.

\begin{abstract}
Introduction: Training with simulators for video-surgery has been proposed as teaching tools, allowing formative evaluation of students under more controlled and safe conditions. Objective: To validate structured program of endosuture training for surgical residents, through two structured scales of acquisition of laparoscopic skills and participant's perception. Methods: This was a quantitative longitudinal experimental study involving 12 residents of surgery from four different hospitals. The training consisted of making endorsements with video simulators in seven sessions over a period of seven months. The evaluation of the progression of the technical competence of the students was carried out by three evaluators, using the Objective Structured Assessmen of Technical Skills (Osats) and the Progression of Proficiency Scale (EPP) in Endorsements. Residents used a Likert scale to evaluate the training program. Anova and chi square tests were performed to compare variables. Correlations and associations between two numerical variables were verified through simple and multiple linear regression, when the determinants were included in addition to the number of training sessions. The Average Ranking was calculated for Likert scale analysis. Comparisons with $p$ value $\leq 0.05$ were considered significant.This study was approved by the Research Ethics Committee of the institution. Results: There was a significant gain in the acquisition of technical competence by the residents during the course, perceived through the two evaluation instruments used (Osats and EPP). As regards the performance of the residents observed by the evaluators through the Osats, there was a statistically significant improvement in the score in relation to the items: Respect to the tissue ( $p=0.006)$, Time and movement ( $p=0.001)$, Knowledge and handling of instruments $p=$ 0.002), Circulation flow ( $p=0.001)$, Knowledge of the specific procedure $(p=0.001)$, by the chisquare test. In relation to the total number of points by EPP and the mean score in the tests by the modified Osats, the total and the mean score in the tests were significant $(<0.001$ and $<0.001)$, using the Kruskal-Wallis test, over the months training. In the students' perception, the training program contributed to the development of surgical skills, with the best evaluated items being the handling of tweezers and needle holders and the knitting. The worst evaluated items were the hapticity and the gait step. Conclusion: There was a significant gain in skills by the residents of surgery during the seven months of simulated training, demonstrated in two structured assessment instruments and by the residents' perception.
\end{abstract}

Recebido em: 16/9/18

Aceito em: 31/10/18

\section{INTRODUÇÃO}

A maioria dos treinamentos em cirurgia inclui simulação no currículo, principalmente para procedimentos endoscópicos e laparoscópicos ${ }^{1}$. A simulação em laparoscopia tem se mostrado efetiva no treinamento de residentes e manutenção de suas habilidades ${ }^{2,3}$. Resulta em melhora do desempenho em cirurgias reais, com menor tempo cirúrgico e complicações pós-operatórias do que os convencionalmente treinados ${ }^{4,5}$. A melhora da performance ocorre graças a quatro condições: repetição intensa de habilidades, avaliação detalhada da performance, feedback específico e ambiente controlado ${ }^{6}$.

$\mathrm{O}$ treinamento exclusivamente em ambiente real preocupa, pois envolve riscos aos pacientes e retarda a progressão das habilidades cirúrgicas. Entre as dificuldades para a aquisição de habilidades em laparoscopia, destacam-se a perda da sensação de profundidade, a perda da sensação tátil, alterações da coordenação mão-olho e o aparecimento do "efeito fulcro", descrito como instrumentos que se movimentam em um ponto fixo à parede abdominal, causando movimentos inversos paradoxais. A propagação e evolução da cirurgia laparoscópica envolvem um investimento maciço em tecnologia, educação e qualificação $0^{6-8}$.

Moura Júnior ${ }^{9}$ desenvolveu um simulador de cavidade abdominal, denominado EndoSuture Trainning Box (ESTB) ${ }^{\circledR}$, que foi bem avaliado pelos alunos e validado pelos cirurgiões. Trata-se de um instrumento de treinamento, progressão de 
habilidades psicomotoras e manutenção da proficiência, demonstrado por meio da Escala de Progressão de Habilidades e Proficiência em Endossuturas (EPP) ${ }^{9}$. Barreira et al. ${ }^{10}$ desenvolveram um currículo sistematizado para treinamento simulado de gastroenteroanastomose laparoscópica, com avaliação da aquisição de habilidades por meio da medição do tempo para executá-las.

A avaliação das habilidades pela Objective Structured Assessment of Technical Skills (Osats) apresenta alta confiabilidade entre residentes de cirurgia geral e cirurgiões, medindo efetivamente em larga escala a capacidade técnica fora do centro cirúrgico, usando o modelo de simulações de bancada ${ }^{11-13}$. Pode avaliar também o desempenho cirúrgico real dentro da sala de operação ${ }^{14-16}$.

Este estudo objetivou avaliar, em residentes de cirurgia, a progressão de habilidades laparoscópicas em treinamento estruturado durante sete meses, por meio dos instrumentos Osats modificado e EPP, além da percepção sobre o programa realizado.

\section{METODOLOGIA}

O treinamento estruturado simulado em endossutura foi desenvolvido no laboratório de habilidades cirúrgicas do Centro Universitário Christus, em Fortaleza (CE), Brasil, utilizando o modelo de videocirurgia EndoSuture Trainning Box - ESTB®9

A coleta de dados ocorreu em sete ocasiões, mensalmente, de outubro de 2016 a abril de 2017, por quatro instrutores, todos cirurgiões seniores em procedimentos videocirúrgicos avançados. Estes se submeteram a um treinamento para compreensão das ferramentas e padronização na avaliação. Doze residentes de cirurgia - sete do primeiro ano, dois do segundo e três do terceiro ano - de quatro hospitais distintos participaram deste programa. Dez dos participantes eram do sexo masculino. Os participantes se apresentaram como voluntários, tendo assinado o Termo de Consentimento Livre e Esclarecido. Não houve evasão de alunos ao longo do treinamento.

Os seguintes materiais foram usados no laboratório de habilidades: simuladores de cavidade abdominal, instrumental cirúrgico, fios, peças de borracha e outros materiais. Após uma primeira sessão teórica composta por um curso básico de endossuturas, vídeos e orientações, os residentes iniciaram o treinamento de habilidades como estereotaxia, triangulação, movimentos invertidos (efeito fulcral), ambidestria, hapticidade, execução de nós e suturas videocirúrgicas (Quadro 1). Esse treinamento de endossuturas no simulador foi repetido durante duas horas, mensalmente, durante sete meses.

\begin{tabular}{|c|c|}
\hline \multicolumn{2}{|c|}{$\begin{array}{c}\text { QUADRo } 1 \\
\text { Etapas do treinamento simulado em } \\
\text { habilidades de endossuturas }\end{array}$} \\
\hline Explanação téorico-prática & $\begin{array}{l}\text { Fundamentos da videocirurgia } \\
\text { Diferenças entre cirurgia convencional e } \\
\text { videocirurgia } \\
\text { Papel do laboratório de habilidades } \\
\text { cirúrgicas no ensino } \\
\text { Introdução de conceitos e habilidades } \\
\text { Demonstração das etapas do } \\
\text { treinamento } \\
\text { Sessão de vídeos cirúrgicos sobre o } \\
\text { modelo de ensino } \\
\text { Visita ao laboratório de habilidades } \\
\text { cirúrgicas }\end{array}$ \\
\hline $\begin{array}{l}\text { Treinamento de } \\
\text { habilidades psicomotoras }\end{array}$ & $\begin{array}{l}\text { Ergonomia } \\
\text { Manuseio de pinças laparoscópicas } \\
\text { Estereotaxia } \\
\text { Triangulação } \\
\text { Efeito fulcral } \\
\text { Ambidestria } \\
\text { Hapticidade } \\
\text { Passada de alças } \\
\text { Passada de fio } \\
\text { Nós ajustados } \\
\text { Endossuturas }\end{array}$ \\
\hline Transferência de objetos & $\begin{array}{l}\text { Com a mão direita para frente } \\
\text { (aheadhand) } \\
\text { Com a mão direita para trás (backhand) } \\
\text { Com a mão esquerda para frente } \\
\text { (aheadhand) } \\
\text { Com a mão esquerda para trás } \\
\text { (backhand) }\end{array}$ \\
\hline Passada de alças & $\begin{array}{l}\text { Passada anterógrada } \\
\text { Passada retrógrada }\end{array}$ \\
\hline Passada do fio & $\begin{array}{l}\text { Passada direita para esquerda } \\
\text { Passada esquerda para direita }\end{array}$ \\
\hline Confecção de nós & $\begin{array}{l}\text { Pegada no instrumental } \\
\text { Pegada no fio } \\
\text { Tamanho do fio } \\
\text { Pegada na agulha } \\
\text { Passada da agulha e fio cirúrgico } \\
\text { Nós cirúrgicos ajustados: formato letra } \\
\text { C e letra D }\end{array}$ \\
\hline Confecção de suturas & $\begin{array}{l}\text { Distância entre pontos } \\
\text { Margem tecidual dos pontos } \\
\text { Simetria das bordas } \\
\text { Tensão dos pontos }\end{array}$ \\
\hline
\end{tabular}

Todos os alunos foram avaliados pelos instrutores por meio de dois instrumentos: EPP e Osats modificado (Quadros 2 e 3, respectivamente), a cada módulo mensal para análise de sua progressão. No final de cada sessão de duas horas de treinamento, o aluno realizava uma sutura com seis pontos com cinco nós ajustados. Em cada encontro houve o acompanhamento de um instrutor, que forneceu feedback apreciativo ao observar os participantes. 


\begin{tabular}{|c|c|}
\hline $\begin{array}{r}\text { QuAD } \\
\text { Escala de Progressã } \\
\text { Proficiência em En }\end{array}$ & $\begin{array}{l}\text { Habilidades e } \\
\text { suturas (EPP) }\end{array}$ \\
\hline Número de pontos em 18 minutos & Classificação \\
\hline 1,2 ou 3 & Insuficiente \\
\hline 4 ou 5 & Regular \\
\hline 6 (entre 15 - 18 min.) & Bom \\
\hline 6 (entre 12 - 15 min.) & Ótimo \\
\hline 6 (abaixo de 12 min.) & Excelente \\
\hline
\end{tabular}

A EPP consiste em avaliar o aluno por sua capacidade de realizar, no tempo de 18 minutos, seis pontos com cinco nós, o que corresponde à quantidade de pontos realizados em uma fundoplicatura tipo Nissen?.

A ferramenta Osats avalia o desempenho das habilidades cirúrgicas, contendo seis itens: respeito ao tecido, tempo e movimento, conhecimento e manuseio dos instrumentos, fluxo da cirurgia, uso dos assistentes e conhecimento do procedi- mento específico ${ }^{11}$. Neste estudo, o item "uso de assistentes" não foi utilizado, visto que não foi permitido aos alunos recorrer a esse tipo de ajuda. Desta forma, consideramos usar o termo Osats modificado.

Ao término do treinamento, os residentes responderam a um questionário com escala Likert de cinco pontos, quanto à aquisição de habilidades específicas proporcionada pelo curso, tais como manuseio de pinças, estereotaxia, triangulação, movimento invertido, hapticidade, ambidestria, passada de alça e de fio, confecção de nós e de endossuturas. O ambiente de estudo e a atuação dos monitores foram também avaliados. Verificou-se a concordância ou discordância das questões avaliadas, por meio da obtenção do ranking médio (RM) da pontuação atribuída às respostas, relacionando à frequência das respostas dos respondentes que fizeram tal atribuição, onde valores menores que 3 são considerados discordantes, e maiores que 3, como concordantes, considerando uma escala de 5 pontos. O valor exatamente 3 seria considerado "indiferente", sendo o "ponto neutro". Para o cálculo do RM, utilizou-se o

\begin{tabular}{|c|c|c|c|c|c|}
\hline \multicolumn{6}{|c|}{ QUADRO 3} \\
\hline & $1^{*}$ & $2 *$ & $3 *$ & $4^{*}$ & $5 *$ \\
\hline Respeito ao tecido & $\begin{array}{c}\text { Usa frequentemente força } \\
\text { desnecessária sobre o tecido ou } \\
\text { causa danos por inadequado uso } \\
\text { de instrumentos. }\end{array}$ & & $\begin{array}{l}\text { Manipula cuidadosamente } \\
\text { tecidos, mas ocasionalmente } \\
\text { causa danos inadvertidos. }\end{array}$ & & $\begin{array}{c}\text { Consistentemente trata de forma } \\
\text { adequada os tecidos, com danos } \\
\text { mínimos. }\end{array}$ \\
\hline & 1 & 2 & 3 & 4 & 5 \\
\hline Tempo e movimento & $\begin{array}{l}\text { Muitos movimentos } \\
\text { desnecessários. }\end{array}$ & & $\begin{array}{l}\text { Tempo/movimentos eficientes, } \\
\text { mas alguns movimentos } \\
\text { desnecessários. }\end{array}$ & & $\begin{array}{l}\text { Evidente economia de } \\
\text { movimentos e máxima eficiência }\end{array}$ \\
\hline \multirow{2}{*}{$\begin{array}{l}\text { Conhecimento } \\
\text { e manuseio dos } \\
\text { instrumentos }\end{array}$} & 1 & 2 & 3 & 4 & 5 \\
\hline & $\begin{array}{l}\text { Falta de conhecimento dos } \\
\text { instrumentos. }\end{array}$ & & $\begin{array}{l}\text { Uso competente de instrumentos, } \\
\text { mas ocasionalmente inadequado. }\end{array}$ & & $\begin{array}{l}\text { Óbvia familiaridade com os } \\
\text { instrumentos }\end{array}$ \\
\hline \multirow[b]{2}{*}{ Fluxo da cirurgia } & 1 & 2 & 3 & 4 & 5 \\
\hline & $\begin{array}{l}\text { Frequentemente parou o } \\
\text { procedimento e demonstrou-se } \\
\text { inseguro do próximo movimento. }\end{array}$ & & $\begin{array}{l}\text { Demonstrou algum planejamento } \\
\text { nos passos a seguir, com } \\
\text { progressão razoável do } \\
\text { procedimento. }\end{array}$ & & $\begin{array}{l}\text { Claramente planejado o curso do } \\
\text { procedimento, com fluxo natural } \\
\text { de um movimento ao outro }\end{array}$ \\
\hline \multirow[b]{2}{*}{ Uso dos assistentes } & 1 & 2 & 3 & 4 & 5 \\
\hline & $\begin{array}{l}\text { Sistematicamente usa mal seus } \\
\text { assistentes ou falha na sua } \\
\text { utilização. }\end{array}$ & & $\begin{array}{l}\text { Utilização adequada dos } \\
\text { assistentes na maior parte do } \\
\text { tempo. }\end{array}$ & & $\begin{array}{l}\text { Estrategicamente utiliza os } \\
\text { assistentes para melhor vantagem } \\
\text { em todos os movimentos }\end{array}$ \\
\hline \multirow[b]{2}{*}{$\begin{array}{l}\text { Conhecimento do } \\
\text { procedimento específico }\end{array}$} & 1 & 2 & 3 & 4 & 5 \\
\hline & $\begin{array}{l}\text { Conhecimento deficiente. Faz-se } \\
\text { necessária instrução na maioria } \\
\text { das etapas. }\end{array}$ & & $\begin{array}{l}\text { Conhece todas as etapas } \\
\text { importantes do procedimento. }\end{array}$ & & $\begin{array}{l}\text { Demonstra familiaridade com } \\
\text { todos os aspectos da operação }\end{array}$ \\
\hline \multicolumn{6}{|l|}{ Total } \\
\hline $\begin{array}{l}\text { Residente habilitado } \\
\text { para realizar o } \\
\text { procedimento } \\
\text { autonomamente. }\end{array}$ & Não & & Talvez & & Sim \\
\hline
\end{tabular}


método de análise de escala do tipo Likert apresentado por Malhotra ${ }^{17}$.

Os resultados quantitativos categóricos foram apresentados em forma de percentuais e contagens, e os numéricos em forma de medidas de tendência central. Foram realizados testes de normalidade para as variáveis numéricas. Foram feitos os testes Anova, Qui-Quadrado e Kruskal-Wallis para comparação entre variáveis, conforme adequado. Foram realizadas regressões lineares simples e múltiplas análises de variância para verificar a significância estatística pelo coeficiente de correlação de Pearson. Foram consideradas significativas as comparações com valor de $\mathrm{p} \leq 0,05$. Os dados foram tabulados e analisados pelo software SPSS, v23, SPSS, Inc. para análise e avaliação dos dados obtidos na coleta.

Este estudo foi aprovado pelo Comitê de Ética em Pesquisa do Centro Universitário Christus, sob o n ${ }^{\circ} 135 / 16$ e protocolo do CEP 62572516.5.00005049.

\section{RESULTADOS}

Os residentes obtiveram uma frequência de participação no curso em torno de $80 \%$. Metade deles afirmou praticar algum instrumento musical ou ter prática com jogos eletrônicos. Com relação à experiência em cirurgias por vídeo, quatro (33,3\%) relataram experiência em videocirurgia como cirurgião. A prática de instrumento musical ou de jogos eletrônicos $(p=0,022)$ sugeriu correlação com a performance geral. Ter experiência em vídeo como cirurgião não pareceu influenciar o desempenho.

Em relação ao número total de pontos pela EPP e à nota média nos testes pelo Osats modificado, o desempenho dos residentes observado pelos avaliadores revelou melhora estatisticamente significante na pontuação no decorrer do tempo $(<0,001)$, utilizando-se o teste Kruskal-Wallis (Tabela 1). Observou-se que o coeficiente de correlação de Pearson, que mede o grau de relacionamento linear entre as duas variáveis quantitativas, mostrou uma variação $R^{2}$ de 0,313 a 0,490 para os critérios avaliados, que representam os itens na escala de avaliação estruturada Osats modificado (Gráfico 1) e número de pontos executados. Estes itens apresentaram relação estatisticamente significante $(\mathrm{p} \leq 0,001)$.

\section{GrÁfico 1}

Média da pontuação da avaliação estruturada OSATS modificado ao longo do treinamento

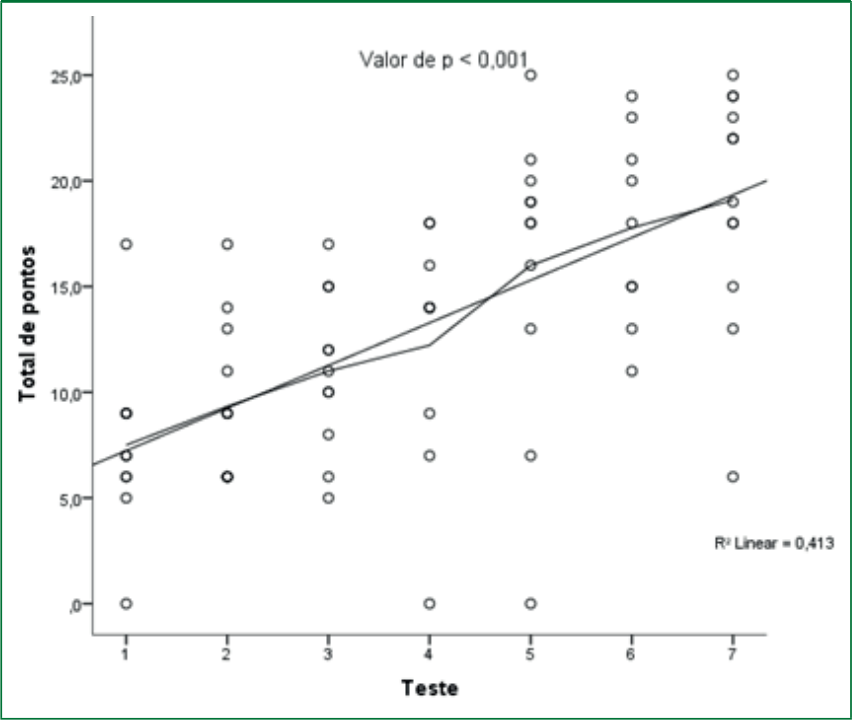

Quanto às respostas dos residentes ao questionário na escala Likert de cinco pontos, ao término do treinamento a maioria dos participantes considerou o treinamento satisfatório, com notas 4 ou 5 nos itens avaliados (aquisição de habilidades, ambiente de trabalho, ergonomia, manuseio de pinças, estereotaxia, triangulação, efeito fulcral, hapticidade, ambidestria, passada de alças, passada de fio, confecção de nós e de endossuturas). $\mathrm{O}$ ambiente de estudo e a atuação dos monitores também foram bem avaliados. Houve percepção de aprendizagem satisfatória pelos alunos.

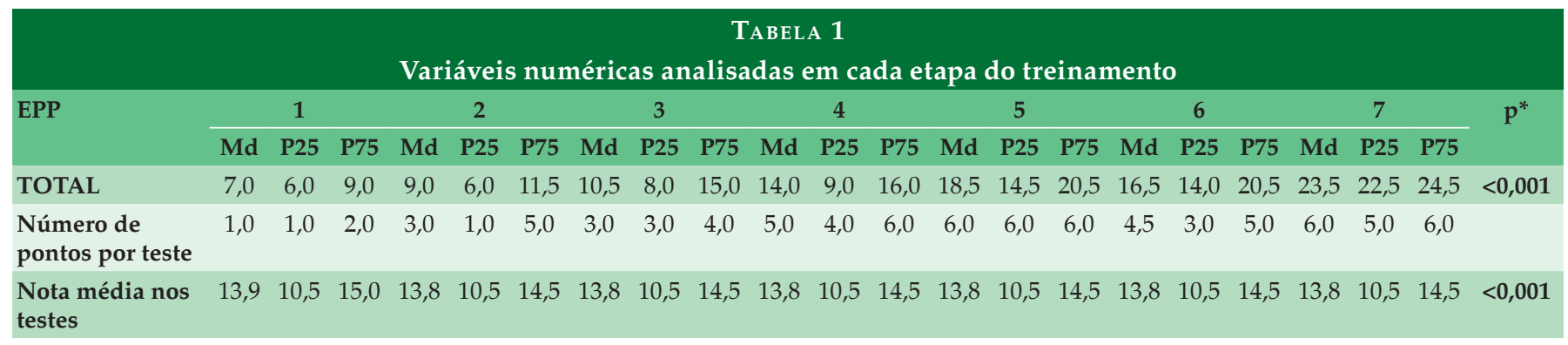




\section{DISCUSSÃO}

O programa estruturado de treinamento de endossuturas desenvolveu atividades teórico-práticas laboratoriais de videocirurgia em modelo de simulação de cavidade abdominal. A frequência de participação nas sete etapas do curso foi elevada, permitindo manter todos os alunos até o final do estudo. São necessárias oito repetições para alcançar a parte superior da curva de aprendizado para sutura laparoscópica ${ }^{18}$. Todos os participantes do estudo cursavam residência médica em cirurgia, com experiência prévia como auxiliares em videocirurgia principalmente.

O instrumento Osats modificado evidenciou progressão dos residentes na aquisição de habilidades para realização de pontos e nós, bem como evolução do número de pontos cirúrgicos realizados em cada teste, confirmada pela EPP, de modo similar a outros estudos ${ }^{2,19}$.

Em estudos prévios com o EndoSutureTrainning Box $E S T B \circledR$, verificou-se uma evolução ascendente do número de pontos executados em determinado tempo ao longo da progressão dos testes ${ }^{9,20}$. Constatou-se evolução do número de pontos e nós ao longo dos meses em estudo, que foi acompanhada progressiva e comparativamente com a avaliação pelo Osats modificado aplicado neste estudo, evitando, assim, perda da qualidade de execução das endossuturas confeccionadas em menor intervalo de tempo.

Duas décadas após a criação do Osats, esta ferramenta validada continua sendo utilizada como método de avaliação em muitas instituições de ensino de residência e em cursos de treinamento simulado em videocirurgia. $\mathrm{O}$ tempo de execução das tarefas, o índice de falhas em cirurgia e a aquisição de habilidades (desempenho) mostraram-se melhores entre estudantes de Medicina e residentes após treinamento simulado em cirurgia laparoscópica ${ }^{21,22}$. Há evidências conflitantes, no entanto, para apoiar a transferência de competências de um ambiente simulado para o centro cirúrgico ${ }^{12,23}$.

As características sociodemográficas apresentaram associação positiva com o desempenho no item relativo à prática de instrumento musical ou jogos eletrônicos neste estudo. Já o fato de ter experiência em videolaparoscopia como cirurgião não influenciou o desempenho. Vale a pena ressaltar o pequeno número de indivíduos alunos neste estudo. $\mathrm{O}$ treinamento simulado proporciona melhoria de habilidades independentemente do ano de residência médica ${ }^{11}$, mas os residentes com maior aptidão (habilidade visual-espacial, percepção de profundidade e capacidade psicomotora) demonstram curva de aprendizado mais rápida e podem atingir a proficiência em uma taxa mais rápida ${ }^{23}$.
Os instrumentos de avaliação estruturados, como o Osats, têm sido úteis ainda em orientar mudanças curriculares, além de identificar necessidades individualizadas de aprendizagem. A cada etapa do treinamento, um cirurgião experiente fornecia feedback individualizado aos participantes, com planejamento de meios para melhorar o desempenho ${ }^{24}$. Isso mostra uma associação com um aprendizado mais rápido e eficaz e, em última instância, permite melhorar a qualidade do atendimento ao paciente ${ }^{23,25}$.

Quanto à percepção da aprendizagem pelos participantes do programa de treinamento, a maioria considerou o treinamento satisfatório, o que foi concordante com o desempenho observado pelos avaliadores ao longo dos sete meses de treinamento. Observou-se ainda elevada satisfação com os facilitadores e com o ambiente de trabalho, sem estresse e sem pressão psicológica. Além disso, não se observou evasão em sete meses. $\mathrm{O}$ uso de simuladores do tipo caixa-preta promove aumento da confiança para os residentes ${ }^{26}$.

A sastisfação com o treinamento e a avaliação de aprendizagem são importantes resultados em avaliação de programas, mas há outros indicadores de qualidade não avaliados aqui, como, por exemplo, a mudança de comportamento e impacto resultante para a saúde. $\mathrm{O}$ currículo proposto para o treinamento deve ser estruturado, com objetivos de aprendizagem bem definidos e avaliação formativa de desempenho dos alunos e do programa em $\mathrm{si}^{27}$.

Uma das limitações do estudo foi a dificuldade de treinar mais avaliadores especialistas voluntários, com participação de apenas três. A uniformidade na avaliação dos alunos é fundamental à credibilidade e validade dos resultados, com aplicação dos mesmos critérios de qualidade para alcançar resultados satisfatórios. Eventuais ausências no curso, mesmo que raras, limitaram o quantitativo nas avaliações. O processo de avaliação implica, de qualquer forma, uma visão reduzida da realidade ${ }^{28}$. Houve progressão de habilidades ao longo dos sete meses de estudo, mas a manutenção da aprendizagem além desse período não foi avaliada.

A implementação de metodologias ativas com a utilização de simuladores de cavidade abdominal tem sido estimulada em centros de treinamento em cirurgia como proposta para reduzir os riscos ao paciente. $\mathrm{O}$ treinamento em realidade virtual tem sido um complemento efetivo na formação de cirurgiões quando se avalia o desempenho cirúrgico ${ }^{21,28}$. A reflexão sobre sua prática após avaliações estruturadas do desempenho permite que médicos em formação melhorem a percepção sobre seu aprendizado. 


\section{CONCLUSÃO}

Um treinamento estruturado para a simulação de endossuturas com sete sessões mensais demonstrou progressão na aquisição de habilidades laparoscópicas por meio dos instrumentos Osats modificado e EPP entre residentes de cirurgia. Os participantes tiveram percepção de aprendizagem adequada em videocirurgias em modelo de bancada.

\section{REFERÊNCIAS}

1. Sroka G, Feldman LS, Vassiliou MC, Kaneva PA, Fayez R, Fried GM. Fundamentals of laparoscopic surgery simulator training to proficiency improves laparoscopic performance in the operating room-a randomized controlled trial. Am J Surg. 2010;199(1):115-20.

2. Aggarwal R, Ward J, Balasundaram I, Sains P, Athanasiou T, Darzi A.Proving the effectiveness of virtual reality simulation for training in laparoscopic surgery. Ann Surg 2007; 246(5):771-779.

3. Palter VN, Orzech N, Reznick RK, Grantcharov TP. Validation of a structured training and assessment curriculum for technical skill acquisition in minimally invasive surgery: a randomized controlled trial. Ann Surg. 2013;257(2):224-30.

4. Zendejas B, Hernandez-Irizarry R, Farley DR. Does simulation training improve outcomes in laparoscopic procedures? AdvSurg 2012; 46:61-71

5. Gurusamy KS, Aggarwal R, Palanivelu L, Davidson BR. Virtual reality training for surgical trainees in laparoscopic surgery. Cochrane Database Syst Rev 2009;21(1):1-4.

6. Ericsson KA. Deliberate practice and the acquisition and maintenance of expert performance in medicine and related domains. Acad Med 2004; 79(suppl): S70-S81.

7. Nacul MP, Cavazzola LT, Melo MC. Situação atual do treinamento de médicos residentes em videocirurgia no Brasil: uma análise crítica. ABCD ArqBrasCirDig. 2015; 28(1):81-85.

8. Stefanidis D, Scerbo MW, Montero PN, Acker CE, Smith WD. Simulator training to automaticity leads to improved skill transfer compared with traditional proficiency-based training: a randomized controlled trial. Ann Surg 2012; 255(1):30-7.

9. Moura Júnior LG. Modelo acadêmico de ensino teórico-prático em vídeo cirurgia, por meio de novo simulador real de cavidade abdominal. Fortaleza, 2015. Doutorado [Tese]- Universidade Federal do Ceará.

10. Barreira MA, Rocha HAL, Mesquita CJG, Borges GCO. Desenvolvimento de um Currículo para Treinamento Simulado de uma anastomose Laparoscópica. RevBrasEduc Med. [online]. 2017;41(3):424-431.
11. Reznick R, Regehr G, MacRae H, Martin J, McCulloch, W. Testing Technical Skill via an Innovative "Bench Station" Examination. Am J Surg. 1997; 173(3):226-30.

12. Niitsu H, Hirabayashi N, Yoshimitsu M, Mimura T, Taomoto J, Sugiyama Y, et al. Using the Objective Structured Assessment of Technical Skills (OSATS) global rating scale to evaluate the skills of surgical trainees in the operating room. Surg Today. 2013;43(3):271-5.

13. Shaharan S, Neary P. Evaluation of surgical training in the era of simulation.World J GastrointestEndosc. 2014;6(9):436-47.

14. Datta V, Bann S, Beard J, Mandalia M, Darzi A. Comparison of bench test evaluations of surgical skill with live operating performance assessments. J Am College Surg. 2004;199(4):603-606.

15. Kundhal PS, GrantcharovTP.Psychomotor performance measured in a virtual environment correlates with technical skills in the operating room. SurgEndosc. 2009;23(3):645-9.

16. Hopmans CJ, den Hoed PT, van der Laan L, van der Harst E, van der Elst M, Mannaerts GH, et al. Assessment of surgery residents'operative skills in the operating theater using a modified Objective Structured Assessment of Technical Skills (OSATS): A prospective multicenter study. Surgery. 2014;156(5):1078-88.

17. Malhotra, N. Pesquisa de Marketing: uma orientação aplicada. Porto Alegre: Bookman, 2001.

18. Botden SM, De Hingh IH, Jakimowicz JJ. Suturing training in augmented reality: gaining proficiency in suturing skills faster. SurgEndosc. 2009; 23(9):2131-2137.

19. Scott DJ, Goova MT, Tesfay ST. A cost-effective proficiency-based knot-tying and suturing curriculum for residency programs. J Surg Res 2007;141(1):7-15.

20. Ferreira Filho F, de Moura Júnior LG, Rocha HAL, Rocha SGMO, Ferreira LFP, Ferreira AFP. Abdominal cavity simulator for skill progression in videolaparoscopic sutures in Brazil. Acta Cir Bras. 2018;33(1):75-85.

21. Chang $\mathrm{OH}$, King LP, Modest AM, Hur HC. Developing an Objective Structured Assessment of Technical Skills for Laparoscopic Suturing and Intracorporeal Knot Tying.J Surg Educ. 2016; 73(2):258-63.

22. Cavalini WLP, Claus CMP, Dimbarre D, Cury Filho AM, Bonin EA, Loureiro MP, et al. Desenvolvimento de habilidades laparoscópicas em estudantes de Medicina sem exposição prévia a treinamento cirúrgico. Einstein (São Paulo). 2014;12(4): 467-472.

23. Buckley CE., Kavanagh DA, Nugent E, Ryan D, Traynor OJ, Neary PC. The impact of aptitude on the learning curve for laparoscopic suturing. The Am J Surg. 2014; 207(2): 263-270. 
24. Ahlborg L, Weurlander M, Hedman L, Nisel H, Lindqvist PG, Felländer-Tsai L, et al. Individualized feedback during simulated laparoscopic training: a mixed methods study. IntJ Med Educ. 2015; 6:93-100.

25. Zevin B, Levy JS, Satava RM, Grantcharov TP. A consensus-based framework for design, validation, and implementation of simulation-based training curricula in surgery. J Am Coll Surg 2012;215(4): 580-586.e3.

26. Supe A, Prabhu R, Harris I, Downing S, Tekian A. Structured Training on Box Trainers for First Year Surgical Residents: does it improve retention of laparoscopic skills? A Randomized Controlled Study.JSurg Educ. 2012;69(5):624-632.

27. Hiemstra E, Schreuder HWR, Stiggelbout AM, Jansen FW. Grading surgical skills curricula and training facilities for minimally invasive surgery. Gynecol Surg. 2013;10(1):63-69.

28. Kasparian AC, Martinez AC, JoverClos RJ, Chércoles RA. Evaluation of technical skills in surgical training. Rev FacCien Med UnivNac Cordoba. 2014;71(3):97-104.

\section{CONTRIBUIÇÃO DOS AUTORES}

Barreto R.M.S., Rocha H.A.L., Borges G.C.O., Peixoto Júnior A. A., Moura Júnior L.G., Peixoto R.A.C. contribuíram com projeto e interpretação dos dados, redação do artigo, revisão crítica do conteúdo intelectual e aprovação final da versão a ser publicada.

\section{CONFLITO DE INTERESSES}

Os autores não têm conflitos de interesse.

\section{ENDEREÇO PARA CORRESPONDÊNCIA}

Raquel Autran Coelho. Rua Silva Jatahy, 1245, Apto 901, Meireles

Fortaleza-CE, Brasil. CEP: 60165070

E-mail: raquelautrancp@gmail.com 